\title{
Distribution of tetracycline in the conjunctiva of patients on long term systemic doses
}

\author{
P N DILLY'AND I A MACKIE? \\ From the 'Department of Anatomy, St George's Hospital Medical School, London; and the \\ 'Department of Ophthalmology, St George's Hospital, London
}

SUMmARY After oral administration of tetracycline fluorescence has been detected in biopsy specimens taken from human and animal conjunctiva. The fluorescence is not uniform but appears to be concentrated in the goblet cells, around the blood vessels, and in a thin film on the external surface of the epithelium. Immersion studies suggest there is a selective binding to the surface of the conjunctiva.

The last decade has seen the increasing use of systemic tetracycline in ophthalmology. It is used in a maintenance dose of $500 \mathrm{mg}$ or $250 \mathrm{mg}$ a day and often without current topical ocular medication. Therapy is often continued over many months and may be maintained for an indefinite period in sight threatening conditions. It usually takes a number of weeks for the effects of treatment to be seen.

'Oral tetracycline is the cornerstone of therapy in acne rosacea' states Wearing.' Its use in ocular rosacea was reported by Marmion as early as $1969,{ }^{2}$ but further reports on its use in this condition did not appear until 1978. ${ }^{3}$ Its other obvious use is in chronic staphylococcal blepharoconjunctivitis, ${ }^{4}$ but it is increasingly being used in allergic and other noninfective diseases of the conjunctiva of a grossly inflammatory nature.

It is interesting that Sneddon ${ }^{5}$ writing on a clinical trial of tetracycline in (skin) rosacea in 1966 remarked that 'unheralded by any fanfares, tetracycline as a method of treatment has come into current use in the last few years', although there was 'in fact virtually no published work on the subject.' The same could be said now of its use in ophthalmology.

It probably works either because of its antibacterial properties, or because it has an antiinflammatory action independent of the well known antimicrobial effects, or both. Several reports have shown tetracycline to be effective in skin diseases in which micro-organisms play no part in the patho-

Correspondence to Mr Ian A Mackic, FRCS. 99 Harley Strect, London WIN IDF. genesis. ${ }^{6-9}$ The localisation of systemic tetracycline in the conjunctiva is not known. As a preliminary study to the investigation of the means by which tetracycline might affect the biodynamics of the conjunctiva we have investigated the distribution of tetracycline in the conjunctiva in patients on long term tetracycline for eye disease.

\section{Materials and methods}

After informed consent biopsy specimens were obtained from the lower eyelid conjunctiva of five patients who had received at least $250 \mathrm{mg}$ of oral tetracycline daily for at least one year and from two patients who had never had tetracycline.

The biopsies were obtained by anaesthetising the lower lid with an injection of $2 \%$ lignocaine without adrenaline. A $2 \mathrm{~mm}$ Elliot trephine was used, and the biopsies were taken from the middle of the palpebral conjunctiva of the lower lid. A portion of the tarsal plate was included to give stability to the tissue. Great care is needed to avoid handling the conjunctival surface of the specimen. The specimens were immersed in normal saline and immediately transported to the laboratory and frozen by putting the specimens in a pot of pentane that was floating in a Dewar flask of liquid nitrogen.

Sections were cut at $10 \mu \mathrm{m}$ on a Bright cryostat and mounted on glass slides. The sections were examined with a Zeiss fluorescence microscope with filters of the blue excitation range $450-490 \mathrm{~nm}$. The controls were similarly treated. The photographs were taken with an exposure time of $30 \mathrm{~s}$ with a Kodak Tri-X film which was developed in Kodak HC110 developer. 

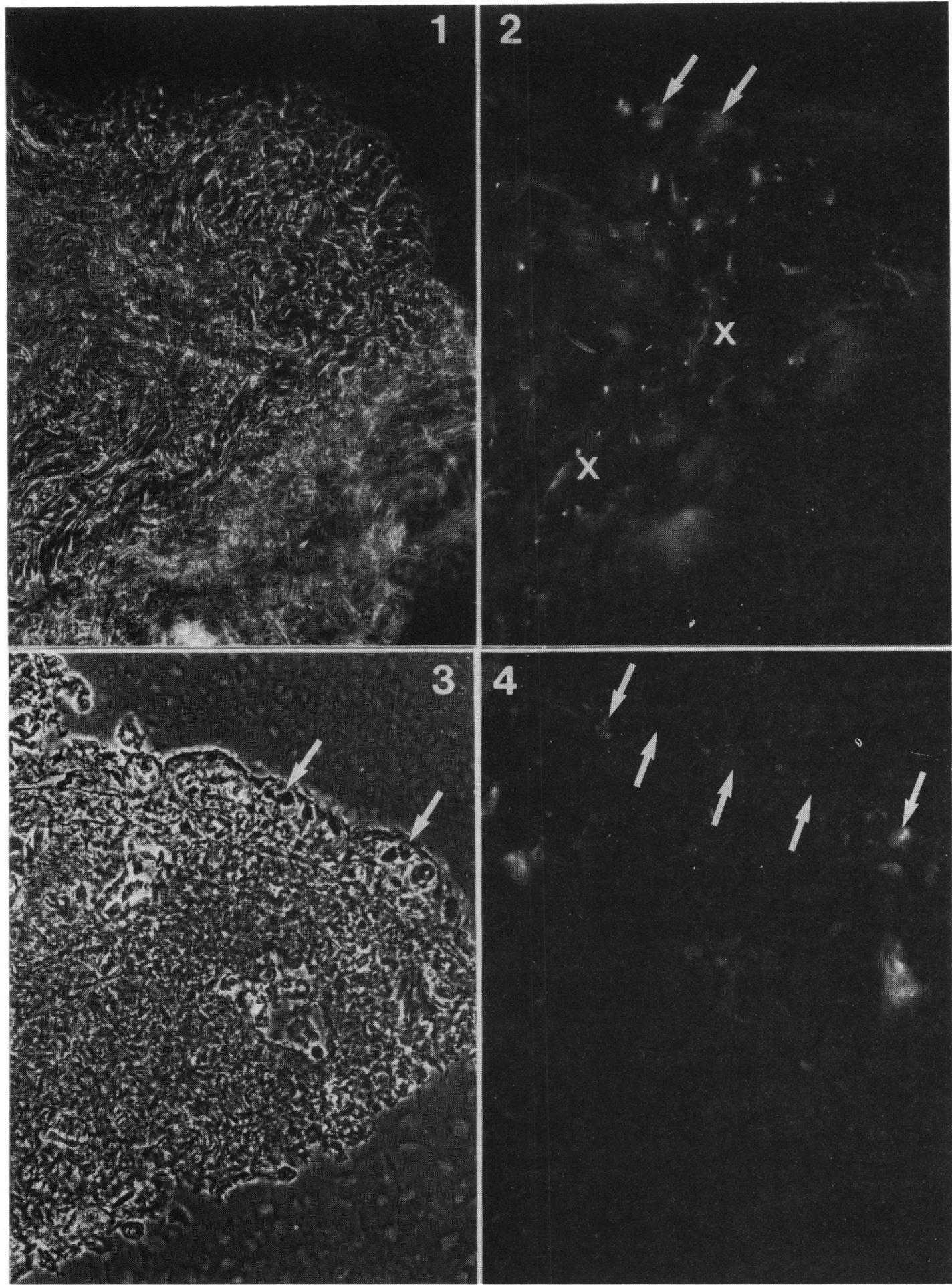
Combined phase contrast microscopy made the handling, orientation, and identification of the sections and recognition of the regions of fluorescence simpler.

Fresh sections of conjunctiva that had not previously been exposed to tetracycline either systemically or locally were immersed in tetracycline eye drops for five hours. The control sections were immersed in saline. They were then washed by gently changing the immersion solution to normal saline. The normal saline was allowed to stand for five minutes before it was replaced. The saline was changed five times before the specimens were examined. Some specimens were left overnight in a large volume of saline that was gently agitated. Fresh post-mortem conjunctivae were also obtained and similarly treated. We could detect no significant difference between the responses of material from the two sources.

The animal material came from guinea-pigs and rats. The tetracycline $(2 \mathrm{mg} / \mathrm{kg})$ was administered daily for periods of up to 100 days.

\section{Results}

Tetracycline fluorescence was detected in the conjunctiva of all the patients who had taken tetracycline orally. There was no fluorescence in the control group.

The fluorescence was not generalised but was restricted to a thin film-like layer on the surface and to small areas in the surface layer of cells. In the deeper layers of the conjunctiva there were also regions showing strong fluorescence (Figs. 1-4).

The surface fluorescence was globular and that of the deeper structures much more linear in overall shape (Figs. 2, 4).

\section{SURFACE FLUORESCENCE}

Control sections of conjunctiva did not autofluoresce. In sections of specimens taken from patients who had taken systemic tetracycline fluorescence occurred all along the surface layers of the conjunctiva, but it was most concentrated in the goblet cells. The goblet cell fluorescence penetrated the whole extent of the cell and both the nucleus and the cytoplasm appeared to fluoresce equally. The general surface fluorescence, however, was only a simple narrow line rather like a membrane some 0.5 $\mu \mathrm{m}$ thick.

In the deeper layers of the stroma the fluorescence was patchy and usually linear in outline. The fluorescence was of a similar intensity to that found in the surface region. Sometimes the fluorescence was in the form of a circular profile. Phase contrast microscopy revealed that these regions contained blood vessels, and that the fluorescence occurred in the areas surrounding blood vessels. This fluorescence around blood vessels resembled that reported from the skin of rats on high doses of systemic tetracycline. ${ }^{10}$

The more linear regions of fluorescence in these deeper layers are associated either with blood vessels that have been sectioned longitudinally or with the inner region of the epithelial cell layers. Sometimes, however, it is not possible to see with the phase contrast microscope any significant morphological difference between those cells that show fluorescence and the surrounding cells (Figs. 1, 2).

We can say little about the rate of accumulation of tetracycline in the conjunctiva of man. But in our experiments with guinea-pigs and rats it is much slower than in the skin. In a preliminary experiment with guinea-pigs we have found tetracycline given by intraperitoneal injection at $2 \mathrm{mg} / \mathrm{kg}$ body weight to appear in the skin after six days but still be undetectable in the conjunctiva after 26 days. The time scale in rats for tetracycline to appear in the conjunctiva is somewhat shorter, but it can still be detected in the skin before it can be found in the eye.

Sections of human conjunctiva that were immersed in tetracycline eye drops and washed showed fluorescence in the goblet cells and a thin layer along the surface, indicating that the tetracycline was localised in the goblet cells and surface layer (Fig. 4). This fluorescence was not removed by repeated gentle washing, and probably represents a binding of the tetracycline to some component of the mucus

Fig. 1 Phase contrast micrograph of the same section as Fig. 2 for orientation. $(\times 300)$.

Fig. 2 Fluorescence micrograph of the same section as Fig. 1. Some of the goblet cells are indicated (arrows). The linear and punctate deeper fluorescence is indicated by X. The fluorescence is produced by tetracycline in the tissue. The section was taken from a biopsy from a patient who had been taking $250 \mathrm{mg}$ tetracycline orally for over 18 months. $(\times 300)$.

Fig. 3 Phase contrast micrograph of the same section as Fig. 4. Some of the goblet cells in the surface layer are indicated by arrows. $(\times 300)$.

Fig. 4 Fluorescence micrograph of the same section as Fig. 3. The surface layer of fluorescence is well seen as is the binding within goblet cells (arrows). Some deeper structures have also absorbed tetracycline and are fluorescing. Fifty-two-hour immersion in tetracycline eye drops in physiological, isotonic, and isosmotic saline. $(\times 300)$. 
secreted by the goblet cells. Whether or not this role is played by the goblet cells in vivo is not known.

We did not detect any significant fluorescence in the basal layer of the epithelium.

\section{Discussion}

The conjunctiva is an epidermal derivative, and these results demonstrate that it has several similar properties to other epidermal structures in its response to tetracycline. Marks and Davies ${ }^{10}$ demonstrated that the fluorescence produced by tetracycline in skin was best seen in the basal layer of the epidermis, the sebaceous glands, and the hair follicles and was preferentially concentrated in parakeratotic areas. They showed also that it remained detectable in the skin after stopping administration and suggested that there was a preferential uptake in cells with a high rate of replication. They observed that the tetracycline did not just diffuse through the dermis and epidermis but was in some way bound to some cellular structures. They suggested that this binding might play a significant part in its therapeutic role.

We have demonstrated a similar binding in the conjunctiva, and it is probable that similar pharmacological effects occur as those in the skin.

The mode of action is unknown, but it is probably not exclusively antimicrobial, because several of the diseases improved by tetracycline therapy are not caused by bacteria. It has recently been demonstrated that tetracycline may have anti-inflammatory properties " quite apart from its antibacterial action.

The thin layer of fluorescence at the surface of the conjunctiva may represent a surface film of tetracycline secreted by the goblet cells. It is known that tetracycline appears in the skin surface film after oral administration of the drug. ${ }^{12}$ It could, of course, be that this thin layer is the remnant of the tear film all of which contains tetracycline, and that this thin layer has survived processing.

The patchy distribution of tetracycline in the conjunctiva suggests that as in the skin the passage of the drug through the conjunctiva is not just simply by diffusion. The association with blood vessels may be nothing more than a reflection of the fact that the tetracycline is blood borne from the gut. This may be the region at which tetracycline is presented in its highest concentrations to the conjunctiva, and the drug diffuses out from here. The tetracycline can get to the goblet cells in the surface layer of the conjunctiva, either by being secreted on to the surface of the eye by the lacrimal or meibomian glands or, by diffusing through the conjunctival epithelium. When it reaches the goblet cells it must in some way be absorbed and concentrated. The surface film too may arrive either by secretion from associated glands, the direct diffusion on to the surface through the conjunctival epithelium, or, as seems more likely to us, by being secreted by the goblet cells along with their mucus on to the surface.

If the immersion experiment results do indicate binding of tetracycline to the mucus layer in contact with the surface of the eye, then this may be a mechanism whereby the tetracycline is prevented from being washed from the surface of the eye by the flow of tears. The preliminary animal experiment results suggest that tetracycline accumulates more slowly in the conjunctiva than in the skin.

We acknowledge the technical expertise of Miss Celia Cope. Our thanks are owed to Mrs G. Coulton who typed the manuscript.

\section{References}

1 Wearing GO. Acne rosacea. In Fraunfelder FT, Roy FH, eds Current ocular therapy. Philadelphia: Saunders, 1980: 147-8.

2 Marmion UJ. Tetracycline in the treatment of ocular rosacea. Proc $R$ Soc Med 1969; 62: 11-2.

3 Brown SI, Shahinian L Jr. Diagnosis and treatment of ocular rosacea. Ophthalmology 1978; 85: 779-86.

4 Smolin G. Staphylococcal blepharitis. In Frauenfelder FT, Roy FH, eds. Current ocular therapy. Philadelphia: Saunders, 1980: 434-5.

5 Sneddon IB. A clinical trial of tetracycline in rosacea. $\mathrm{Br} J$ Dermatol 1966; 78: 649-53.

6 Piamphongsant $T$. Tetracycline for the treatment of pityriasis lichenoides. Br J Dermatol 1974; 91: 319-22.

7 Chan HL. Panniculitis (Rothmann-Makia) with good response to tetracycline. Br J Dermatol 1975; 92: 351-4.

8 Sneddon IB. The treatment of steroid induced rosacea and perioral dermatitis. Dermatologica 1976; 152: (suppl 1) 231-7.

9 Thomsen K, Osterbye P. Pustulosis palmaris et plantaris. Br J Dermatol 1978; 89: 273-96.

10 Marks R, Davies MJ. The distribution in the skin of systemically administered tetracycline. BrJ Dermatol 1969; 81: 448-51.

11 Elewski BE, Lamb BAJ, Sams WM, Gammon WR. In vivo suppression of neutrophil chemotaxis by systemically and topically administered tetracyclinc. J Am Acad Dermatol 1983; 8: $807-812$.

12 Rashleigh PL, Rife E, Goltz RW. Tetracycline levels in skin surface film after oral administration of tetracycline to normal adults and to patients with acne vulgaris. J Invest Dermatol 1967; 49: $611-5$. 